\title{
The Onset of the Flash Transition in Single Crystals of Cubic Zirconia as a Function of Electric Field and Temperature
}

\author{
D. Yadav and R. Raj \\ Department of Mechanical Engineering \\ University of Colorado Boulder \\ Boulder, CO 80309
}

December 2016

Submitted to: Scripta Materialia (First Revision)

\begin{abstract}
We have measured the onset of flash in cubic zirconia single crystals, in experiments similar to flash sintering. This behavior is compared with results on sintering of porous specimens of 8-10 mol\% cubic zirconia. The single crystals flash at lower temperatures and lower electric fields, which does not support an unalienable role for particle-particle interfaces in the flash mechanism. The flash onset in single crystals occurs at similar values of the power density as the powder samples, which, furthermore, lies in the same range as the onset of the flash transition in several oxides.
\end{abstract}

Corresponding Author: Rishi Raj, rishi.raj@colorado.edu

Key words: flash sintering, single crystals, cubic zirconia

(C) 2017. This manuscript version is made available under the Elsevier user license http://www.elsevier.com/open-access/userlicense/1.0/ 


\section{Introduction}

Flash sintering has been shown to possess three essential characteristics, a highly non-linear rise in conductivity [1], electroluminescence [2] and a colossal rate of diffusional mass transport which produces ultrafast rates of sintering [1]. The rates of sintering have been shown to be three to four orders of magnitude faster than in conventional sintering [3]. For example, yttria stabilized zirconia which nominally requires several hours to densify at $1400-1500{ }^{\circ} \mathrm{C}$, can be sintered in mere seconds at a furnace temperature of $\sim 800{ }^{\circ} \mathrm{C}[1]$, and specimen temperature of $1100-1200{ }^{\circ} \mathrm{C}$ [4,5]. Flash experiments can be carried out at isothermal furnace temperatures [6], where an electric field is applied as a step function, or by applying an electric field and then heating the furnace at a constant rate [1]. Once the flash initiates it is necessary to switch the power supply to current control.

The role of particle-particle interfaces in porous specimens remains of interest in the physics of flash sintering. Local heating at such interfaces $[7,8]$, even melting $[9,10]$ have been proposed as the underlying cause. Therefore, we ask whether or not single crystals, which are devoid of such features, may also exhibit the flash effect. Rather surprisingly, the single crystals flash at lower temperatures, and at lower electrical fields, than powder specimens, which is contrary to the proposal that particle-particle interfaces are the source of flash sintering.

The electrical power density dissipated in the specimen increases abruptly at the onset of the flash. Indeed the value of the power density at the flash transition has become of interest since it has been shown to fall within a narrow range for flash sintering of many different ceramic materials [11]. We examine how far the onset of the flash in single crystals of yttria stabilized cubic zirconia agrees with this behavior.

\section{Experimental}

Single crystals of cubic zirconia ( $8 \mathrm{~mol} \%$ yttria stabilized zirconia) cut into parallelepiped shaped specimens, with a rectangular cross-section, were used. Thin platinum wires, wrapped around the ends of the sample, served as the electrodes, leaving an effective gage length of $4 \mathrm{~mm}$ with a cross section of $2 \mathrm{~mm} \times 0.7 \mathrm{~mm}$. The gage length was aligned along the [100] axis of single crystal. Platinum paste was applied to improve electrical contact between the wire and the sample. The samples were hung with these platinum wires within a furnace (Applied Test System, PA, USA). Electrical power was provided from a 
600W DC power source (Sorensen 300-2, Sorensen, San Diego CA). The current was measured with a digital multi-meter (Keithley 199) connected to a National Instruments GPIB-USB interface. Data was acquired through a graphical-user-interface developed inhouse, with MATLAB.

Electric field was applied at room temperature. It ranged from $50 \mathrm{~V} \mathrm{~cm}^{-1}$ to $490 \mathrm{~V}$

$\mathrm{cm}^{-1}$. The current limit was set to $25 \mathrm{~mA} \mathrm{~mm} \mathrm{~m}^{-2}$ for all experiments. The furnace was heated at a constant rate until the onset of the flash, which was signaled by an abrupt increase in the conductivity of the specimen. This critical value of the temperature depended on the strength of the applied field. The power supply was switched to from voltage to current control upon reaching the current limit. The samples were held in a constant state of flash, briefly, under current before turning off the power supply to the specimen. The power dissipated was calculated from the product of voltage and current and then normalized with respect to the volume of the sample, and expressed in units of $\mathrm{mW} \mathrm{mm}^{-3}$.

\section{Results}

The specimen was suspended within a conventional furnace, the field was applied and the furnace was heated as a rate of $10{ }^{\circ} \mathrm{C} \mathrm{min}^{-1}$, starting from room temperature. When the specimen flashed the power supply was switched to current control, which was held for $\sim 10 \mathrm{~s}$ before the power to the specimen was turned off. A current limit of $25 \mathrm{~mA} \mathrm{~mm}^{-2}$ was used for all experiments. The principal variable in the experiments was the electric field, which was increased from $50 \mathrm{~V} \mathrm{~cm}^{-1}$ up to $490 \mathrm{~cm}^{-1}$, in steps of $20 \mathrm{~V} \mathrm{~cm}^{-1}$.

The voltage and current were measured continuously through the experiment, and their product was used to calculate the power expended in the specimen. These results, showing the change in the power density, and the current density, are given in Fig. 1. The curves show the characteristic flash transition, most clearly seen when the current reaches its preset limit and the experiment is changed from voltage control to current control. At higher fields the transition occurred sooner, that is at lower temperatures and is, apparently, sharper. Indeed, at these low temperatures the transition is so sharp that the power supply, which is stated to switch from voltage to current control in $50 \mathrm{~ms}$, is unable to comply and current overshoots the prescribed limit, albeit only very briefly.

The field and temperature combinations for the transition are plotted in Fig. 2(a). Data for powder specimens from the literature are included in 2(b). They are remarkably 
different. While $2500 \mathrm{~V} \mathrm{~cm}^{-1}$ is required to flash the powder samples near $300{ }^{\circ} \mathrm{C}$, the single crystals flash at a similar temperature at a field of only $490 \mathrm{~V} \mathrm{~cm}^{-1}$. These differences are highlighted in Fig. 2(c). While both sets of data show the same trend, the single crystals are far easier to flash than the powder samples. These results contradict the idea that local heating at particle-particle interfaces are the genesis of the flash phenomenon. It is more likely that the event occurs within the crystal matrix.

In constant heating rate experiments the flash transition is most clearly evident in the Arrhenius plots of the power dissipation. Below the transition the conductivity is expected to be thermally activated. Near the transition the power dissipation in the specimen rises quickly.

Such Arrhenius plots are shown in Fig. 3. Note that despite the wide range of fields and temperature for the onset of the flash, the transition occurs within a narrow band of the power density. Indeed, such behavior has been seen in experiments with various oxides as well [11]. In the present work the transition bifurcates into a low temperature, and a high temperature regime. The low temperature regime persists to a field of about $230 \mathrm{~V} \mathrm{~cm}^{-1}$ and up to $\sim 400{ }^{\circ} \mathrm{C}$ with the data falling within a band of $4-8 \mathrm{~mW} \mathrm{~mm}^{-3}$. The power dissipation falls into a higher band of $20-40 \mathrm{~mW} \mathrm{~mm}^{-3}$ above $500{ }^{\circ} \mathrm{C}$ and at fields from $210 \mathrm{~V} \mathrm{~cm}^{-1}$ down to $50 \mathrm{~V} \mathrm{~cm}^{-1}$. The implication of these results is discussed in the next section.

The self-similarity of the shape of the power transition was explored further. By inspection we note that the transitions become more gradual, that is they are slower, as the field is reduced and the transition temperature rises. We wondered if this could be a result of an internal clock associated with the mechanism of the flash. This hypothesis was explored by compressing the time scale for the data at different temperatures to see if the results could be made to overlap. The result of this exercise is given in Fig. 4. The upper graph in Fig. 4(a) shows the transition for different fields over a time span of $20 \mathrm{~s}$. The time is counted from the instant that the power dissipation reaches a value of $50 \mathrm{mw} \mathrm{mm}^{-3}$. A period of $20 \mathrm{~s}$ from this reference point envelops all the transitions from $230 \mathrm{~V} \mathrm{~cm}^{-1}$ to $490 \mathrm{~V} \mathrm{~cm}^{-1}$. The transition, from this starting point, occurs earlier at higher fields than at lower fields. The curves in Fig. 4(b) show the same data, but where the scale for the slower transitions is compressed until they match the data at the highest field. For example $20 \mathrm{~s}$ time period at $490 \mathrm{Vcm}^{-1}$, where the flash occurs at $315{ }^{\circ} \mathrm{C}$, is equivalent to $7 \mathrm{~s}$ for the same "real time" period at $230 \mathrm{~V} \mathrm{~cm}^{-1}$ which occurs at $415{ }^{\circ} \mathrm{C}$. In other words, $20 \mathrm{~s}$ at $315^{\circ} \mathrm{C}$ is equivalent to $7 \mathrm{~s}$ at $415^{\circ} \mathrm{C}$. Thus it 
would appear that increasing the temperature from $315^{\circ} \mathrm{C}$ to $415^{\circ} \mathrm{C}$ accelerates the intrinsic time clock by a factor of about three, and so on. There may be a role for the electric field in the time-temperature relationship, as well.

The results in Figs 3 and 4 suggest that the flash is instigated at a specific value of the power density. Since the specimen is under voltage control during the transition, the power density is directly related to the specific conductivity by

$$
P_{W}^{*}=\Gamma^{2} \sigma^{*}
$$

where $P_{W}^{*}$ is the power density at the transition, $\Gamma$ is the electric field (under voltage control), and $\sigma^{*}$ is the specific conductivity at the transition temperature, $T^{*}$, which are related by $\sigma^{*}=\sigma_{o} \exp \left(-\frac{Q}{R T^{*}}\right)$, where $\sigma_{o}$ is the pre-exponential, $Q$ is the activation energy, and $T^{*}$ is the transition temperature. Since $P_{W}^{*}$ is found to have an approximately constant value, we obtain the following relationship between the applied field and the transition temperature

$$
\Gamma^{*}=\sqrt{\frac{P_{W}^{*}}{\sigma_{o}}} \exp \left(\frac{Q}{2 R T^{*}}\right)
$$

According to Eq. (2) the electric field should follow an Arrhenius relationship with an activation energy that is one half the value for the specific conductivity. The data are so plotted in Fig. 5, and they do indeed fit the Arrhenius form but with a stepwise shift from low temperature to high temperature behavior. The activation energy, $Q$, for both low and high temperature regimes is $64 \mathrm{~kJ} \mathrm{~mol}^{-1}$. Oxygen ion diffusion measurements over a wide range of conditions have shown the outer bounds of the activation energy for ionic transport to lie between $0.8 \mathrm{eV}$ to $1.2 \mathrm{eV}\left(77-115 \mathrm{~kJ} \mathrm{~mol}^{-1}\right)$ [12]. The difference is not large enough to infer, definitively, but does suggest that electrons could have been responsible for charge transport in our experiments; this point is further discussed in the next section.

\section{Discussion}

The following questions emerge from the current study.

(i) What may be the significance of the activation energy measured in Fig. 5? 
(ii) What is the significance of the fact that the flash transition occurs at a constant value of the power density for powder specimens and single crystals alike?

Finally, we discuss the relevance of recent theories of thermal runaway as the explanation of flash sintering, to the present results.

The activation energy for the specific conductivity, given in Eq. (2), is measured to be $64 \mathrm{~kJ} \mathrm{~mol}^{-1}$, lower than the value for oxygen ion diffusion, which lies within $77-115 \mathrm{~kJ}$ $\mathrm{mol}^{-1}$ [12] It is possible that this difference arises from the charge transport mechanism in flash experiments. There is some evidence for this behavior in two papers in the literature. In a very recent article West and co-workers show that a DC bias applied across yttria stabilized zirconia causes high electronic conductivity at $563{ }^{\circ} \mathrm{C}$, and that this effect becomes increasingly significant as the bias is increased, reaching an electron transport number of 0.8 [13].

In an early paper Wagner et al. [14] report on experiments carried out with electrochemical cells made from cubic zirconia and nickel metal electrodes. When current was passed, a high voltage developed across the cell which quickly declined as a higher state of conductivity was established. Electron microscopy of the interfaces revealed a redox reaction where nickel oxidized into nickel-oxide at one electrode and the electrolyte was reduced and reacted with nickel to form a nickel-zirconium intermetallic at the other electrode. They were able to use the cross-sectional images to calculate the number of oxygen ions transported across electrolyte from the thickness of the nickel-oxide layer. The total charge transported during the experiment was measured by integrating the current with time. It was discovered that oxygen ions could account for only $5 \%$ of the total charge transported through the cell, leading to the conclusion that the electrical current was predominantly electronic.

It is interesting to note, in Fig. 6, that while the $8 \mathrm{~mol} \% \mathrm{YSZ}$ cubic zirconia single crystals flash at lower temperatures than powders of the same composition, the $\mathrm{ZnO}$ single crystals are more difficult to flash than powders [15]. However, the main feature of these results is that $\mathrm{ZnO}$ single crystals and powders flash within the same narrow band of power density, as the single crystals and powder samples of 8 YSZ. While we do not know why the single crystals of $\mathrm{ZnO}$ have a higher electrical resistivity than the powders - impurities and 
doping may have been the reason - it matters only that both reach a certain level of power dissipation in order to flash. If the resistance it higher then the flash temperature is higher as well; if the resistance is lower then the flash temperature is lower.

The Arrhenius plots of power density in Fig. 6 for many oxides point to the unusual result that the onset of the flash occurs in a band of power density, for several oxides including powder samples as well as single crystals. The flash temperatures range from 300 ${ }^{\circ} \mathrm{C}$ to $1300{ }^{\circ} \mathrm{C}$, and the fields from $7.5 \mathrm{~V} \mathrm{~cm}^{-1}$ to $1000 \mathrm{~V} \mathrm{~cm}^{-1}$, yet all oxides show the onset of flash within a narrow band of power density.

The analysis of the onset of flash has been of interest in recent publications $[11,16,17]$. But these papers are concerned not with the onset of the flash as described here, but rather with the theory of thermal runaway for explaining flash sintering, completely. (The present work is not concerned with flash sintering but rather with the onset of flash in powder and single crystals of 8YSZ.) For example in [16] it is stated "Expecting Joule heating to be the likely cause for $\mathrm{T}$ to exceed $\mathrm{T}_{\mathrm{f}}$, we use the following ansatz to seek a possible criterion for the onset of thermal runaway, at $\mathrm{T}=\mathrm{T}_{\mathrm{c}}$ ", and in [17] that "As $\mathrm{T}_{\mathrm{f}}$ reaches $\mathrm{T}$, Joule heating takes over causing T to run away", based upon the competition between electrical power dissipation and black body radiation. Furthermore, these analyses are contradicted in [11] where it is shown that the specimen would evolve into a steady state where Joule heating becomes equal to black body radiation, with the flash onsetting after an incubation time. The issue of thermal runaway, which in any case is not relevant to the current work, remains unresolved.

It is interesting to note that the Arrhenius plots of power density, given in Fig. 3, apparently bifurcate into two regimes. At lower temperatures the transition band lies at lower power density than for the transitions that occur at the higher temperatures. This bifurcation suggests a role for the heat-loss mechanism in the transition. The heat loss at higher temperatures is dominated by black body radiation, which varies as the fourth power of the temperature, while convection and conduction, which scales with a far lower power of temperature, dominates heat loss in the low temperature range. Yet, as noted in [11] the continued Arrhenius increase in electrical conductivity cannot explain the entire flash sintering phenomenon. The activation energy for electrical conductivity obtained from the graphs in Fig. 5 and the discussion above related to [14,15], may suggest a transition to 
electronic conductivity. In-situ measurements are unable to explain the ultrafast sintering rates merely in terms of the rise in specimen temperature [2]. It would appear that the flash transition shown so eloquently in Fig. 6 spells the onset of a state of affairs that we do not yet understand.

\section{Summary}

(i) Single crystals of cubic zirconia flash more easily, that is at lower temperatures and at lower electric fields, than powders during flash sintering experiments (Fig. 2).

(ii) The flash transition is embodied in a constant value of the power density that holds over a wider range of fields and temperatures for powders, single crystals and for several oxides that flash over a wide range of fields and temperatures (Fig. $6)$.

(iii) The transition curves can be made to overlap exactly by normalizing the time scale. For example, the slower transition at higher temperatures can be matched with the quicker transition at lower temperature by compressing the time scale for the slower transition (Fig. 4).

(iv) Measurements of the activation energy for the specific conductivity of zirconia are than for oxygen ion diffusion. This difference may imply that conduction occurs mostly by electrons in the flash-transition regime (Fig. 5).

\section{Acknowledgements}

This research was supported by the Office of Naval Research under Grant No. N00014-15-12505 under the direction and advice of Dr. Antti Makinen.

\section{References}

1. M. Cologna, B. Rashkova, R. Raj, J. Am. Ceram. Soc. 93 (2010) 3556-3559.

2. K. Terauds, J.M. Lebrun, H.H. Lee, T.Y. Jeon, S.H. Lee, J.H. Je, R. Raj, J. Eur. Ceram. Soc. 35 (2015) 3195-3199.

3. R. Raj, J. Eur. Ceram. Soc. 32 (2012) 2293-2301.

4. M. Cologna, A.L.G. Prette, R. Raj, J. Am. Ceram. Soc. 94 (2011) 316-319.

5. J.A. Downs, V.M. Sglavo, J. Am. Ceram. Soc. 96 (2013) 1342-1344. 
6. J.S.C. Francis, R. Raj, J. Am. Ceram. Soc. 9 (2013) 2754-2758.

7. R. Muccillo, E.N.S. Muccillo, J. Electroceram. (2016) DOI:10.1007/s10832-0160054-x.

8.T. Jiang, Z. Wang, J. Zhang, X. Hao, D. Rooney, Y. Liu, W. Sun, J. Qiao, K. Sun, J. Am. Ceram. Soc. 98 (2015) 1717-1723.

9. R. Chaim, Materials. 9 (2016) 280.

10. J. Narayan, Scr. Mater. 69 (2013) 107-111.

11. R. Raj, J. Am. Ceram. Soc. 99 (2016) 3226-3232.

12. M. Kilo, C. Argirusis, G. Borchardt, R.A. Jackson, Phys. Chem. Chem. Phys. 5 (2003) 2219-2224.

13. N. Masó, A.R. West, Chem. Mater. 27 (2015) 1552-1558.

14. T. Wagner, R. Kirchheim, M. Rüehle, Acta Metall. Mater. 40 (1992) S85-S93.

15. Y. Zhang, J.I. Jung, J. Luo, Acta Mater. 94 (2015) 87-100.

16. Y. Dong, I.W. Chen, J. Am. Ceram. Soc. 98 (2015) 2333-2335.

17. Y. Dong, I.W. Chen, J. Am. Ceram. Soc. 98 (2015) 3624-3627.

\section{List of Figures}

Figure 1. (a) Flash transitions in power density, and (b) in current density in constant heating rate experiments. Note the current limit plateau when the power supply switches from voltage to current control. The overshoot at high electric fields arises from the delay in changing to current control, which is $50 \mathrm{~ms}$ according to specifications for the power supply.

Figure 2. The flash transition measured in (a) in single crystals in the current study, and (b) powder samples in a sintering experiment $[4,5]$. (c) Shows the results from powder and single crystal experiments plotted in the same graph to highlight that the single crystals flash at lower temperature and at lower electric fields.

Figure 3. Arrhenius plots of the power density for experiments carried out at fields ranging from $50 \mathrm{~V} \mathrm{~cm}^{-1}$ to $490 \mathrm{~V} \mathrm{~cm}^{-1}$. Note that the transition falls in narrow bands of power density, 
one at high temperature in the $20-40 \mathrm{~mW} \mathrm{~mm}^{-3}$ range, and the other at lower temperatures spanning $4-8 \mathrm{~mW} \mathrm{~mm}^{-3}$.

Figure 4. The flash transition in the $230 \mathrm{~V} \mathrm{~cm}^{-1}$ to $490 \mathrm{~V} \mathrm{~cm}^{-1}$ range shown on an expanded scale of 20 seconds time. (a) Note that the transition becomes more gradual at lower field (or higher flash temperature); it is sharper at $490 \mathrm{~V} \mathrm{~cm}^{-1}$ than at $230 \mathrm{~V} \mathrm{~cm}^{-1}$. (b) The curves are shown to overlap near exactly for the rise in conductivity if the time-scale for the slower transitions is compressed; they then match the transition curve at $490 \mathrm{~V} \mathrm{~cm}^{-1}$.

Figure 5. An Arrhenius plot of the electric field vs. 1/T(K) as suggested by Eq. (2). The slope, which is related to $(\mathrm{Q} / 2)$ gives an activation energy for specific conductivity, $\mathrm{Q}=64 \mathrm{~kJ} \mathrm{~mol}^{-1}$.

Figure 6. An overlay of the flash transition from earlier work [12] and the current results, which support the result that flash non-linearity occurs within a narrow band of power density. 


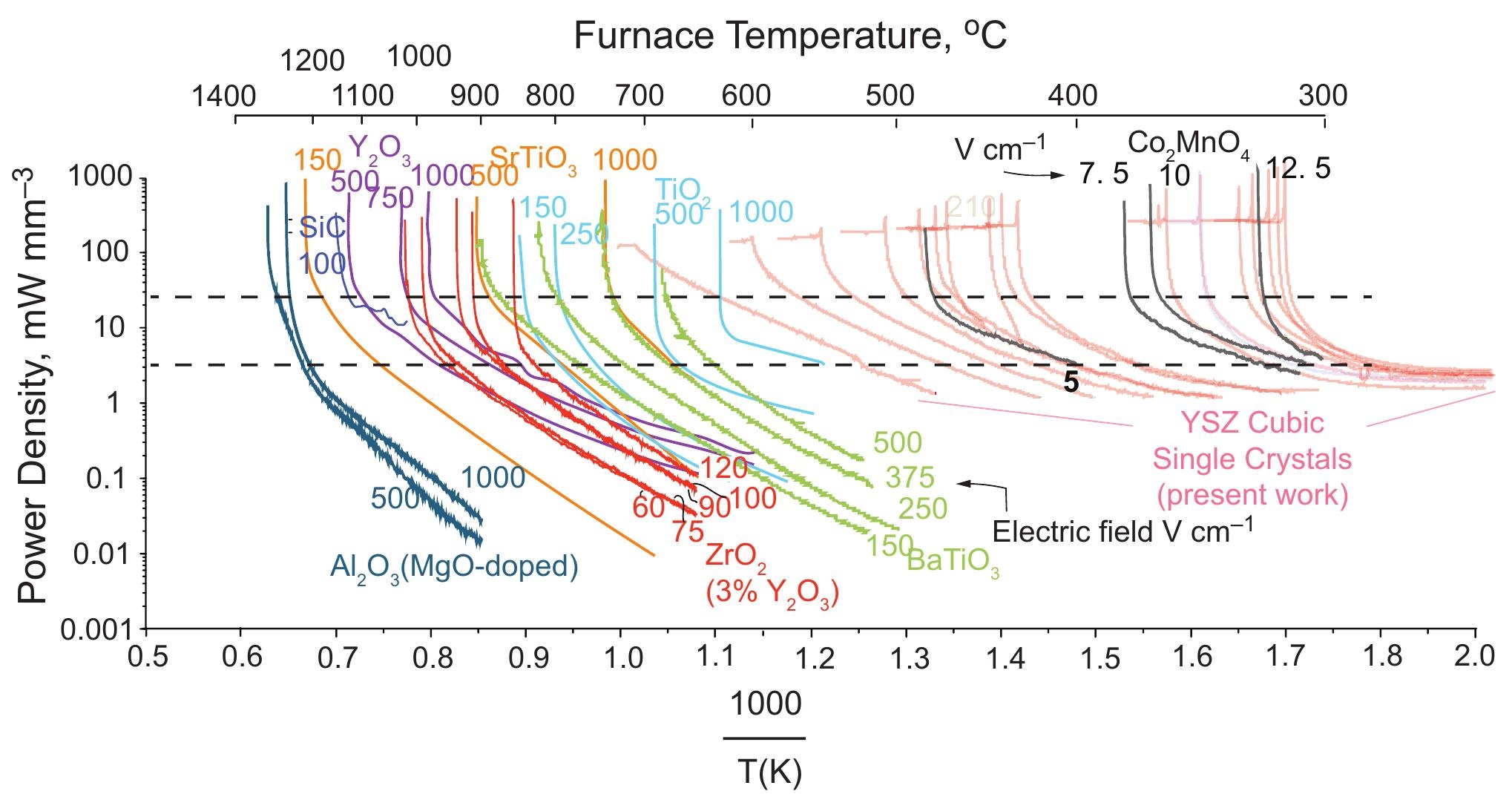



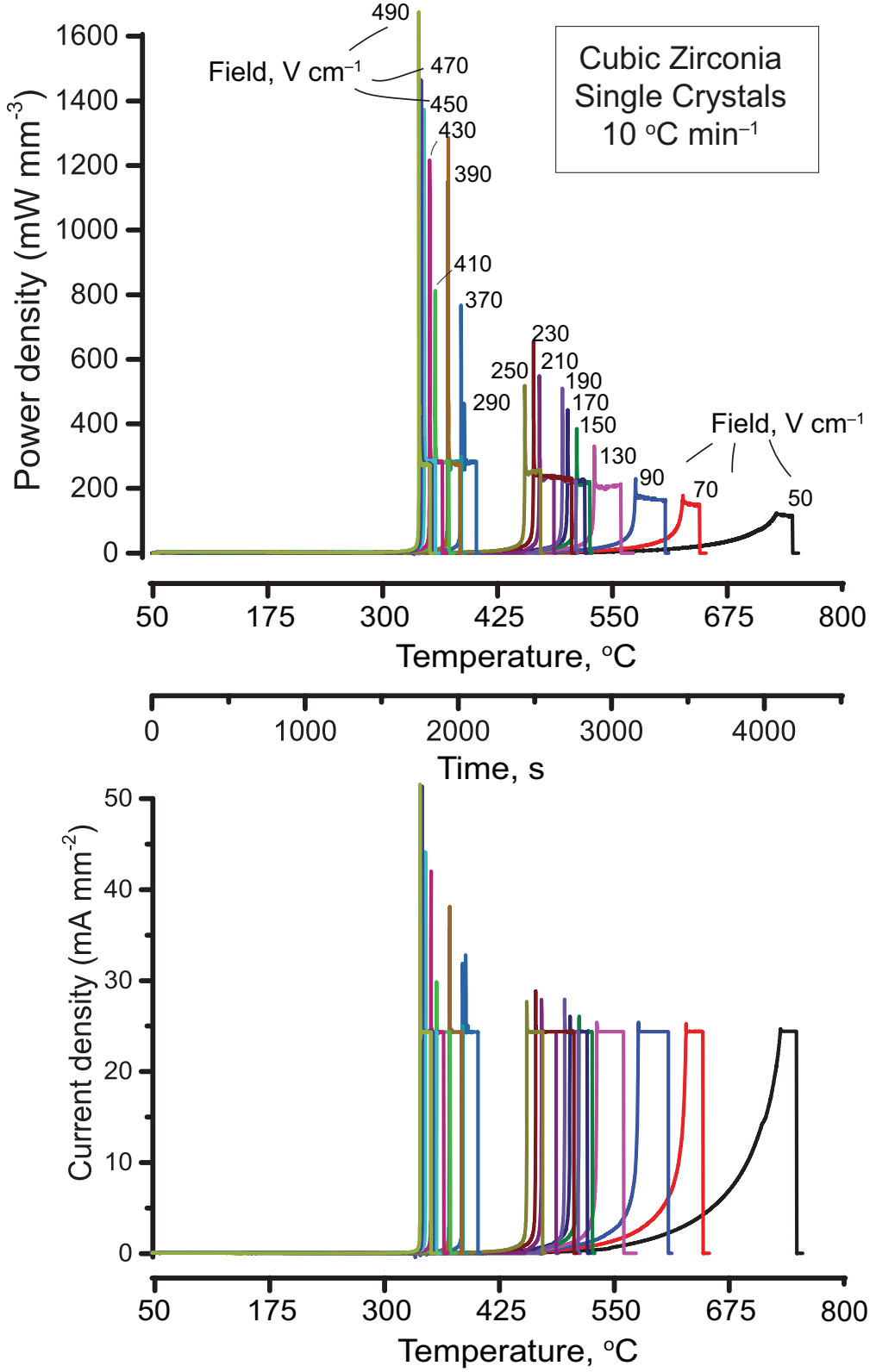

Figure 2. The flash transition measured in (a) in single crystals in the current study, (b) in powder samples in a sintering experiment $[4,5]$. Both sets are data are compared together in (c). 


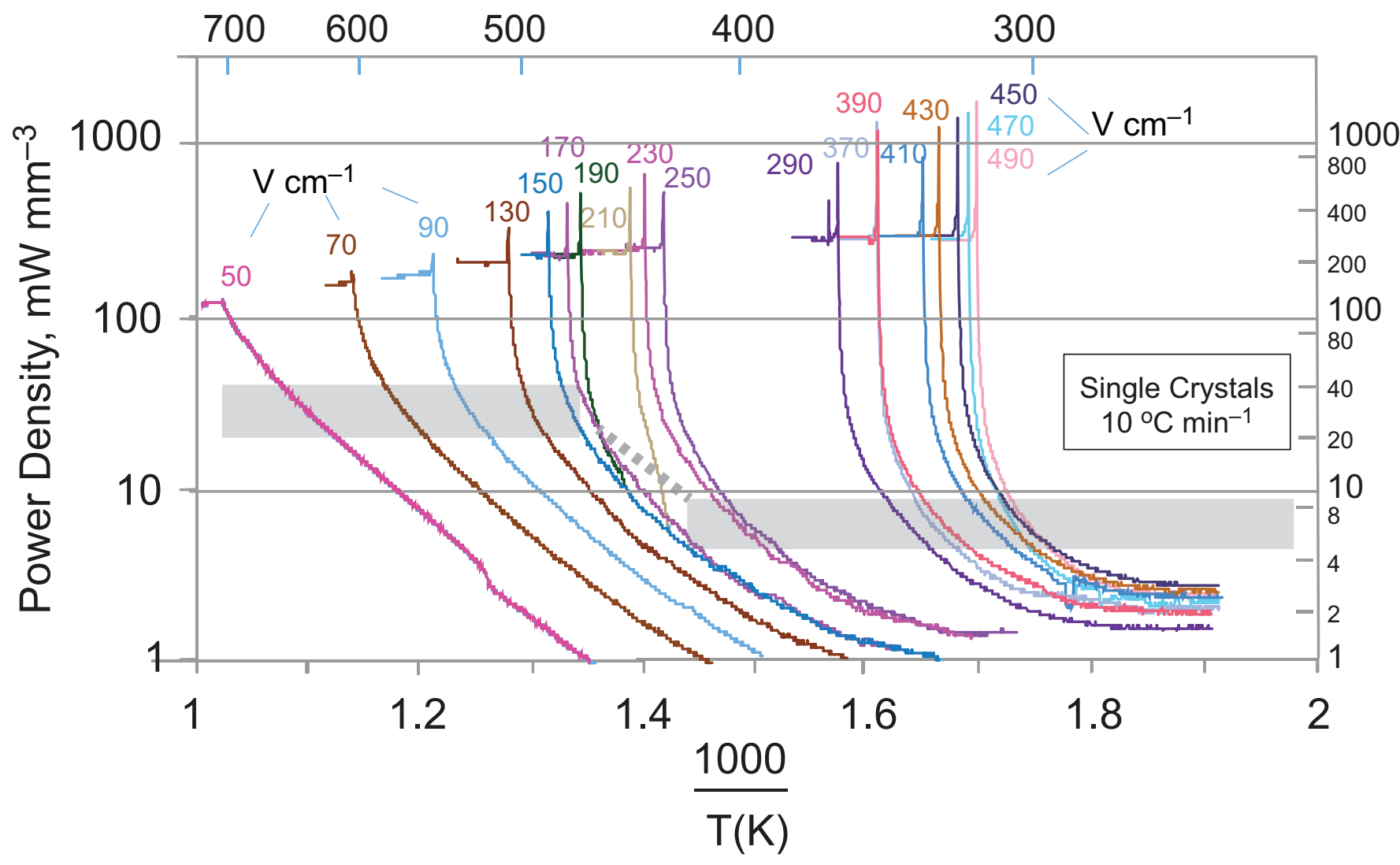

Figure 3. Arrhenius plots of the power density for experiments carried out at fields ranging from $50 \mathrm{~V} \mathrm{~cm}-1$ to $490 \mathrm{~V} \mathrm{~cm}-1$. Note that the transition falls in narrow bands of power density, one at high temperature in the $20-40 \mathrm{~mW}$ $\mathrm{mm}-3$ range, and the other at lower temperatures spanning $4-8 \mathrm{~mW} m \mathrm{~m}-3$. 


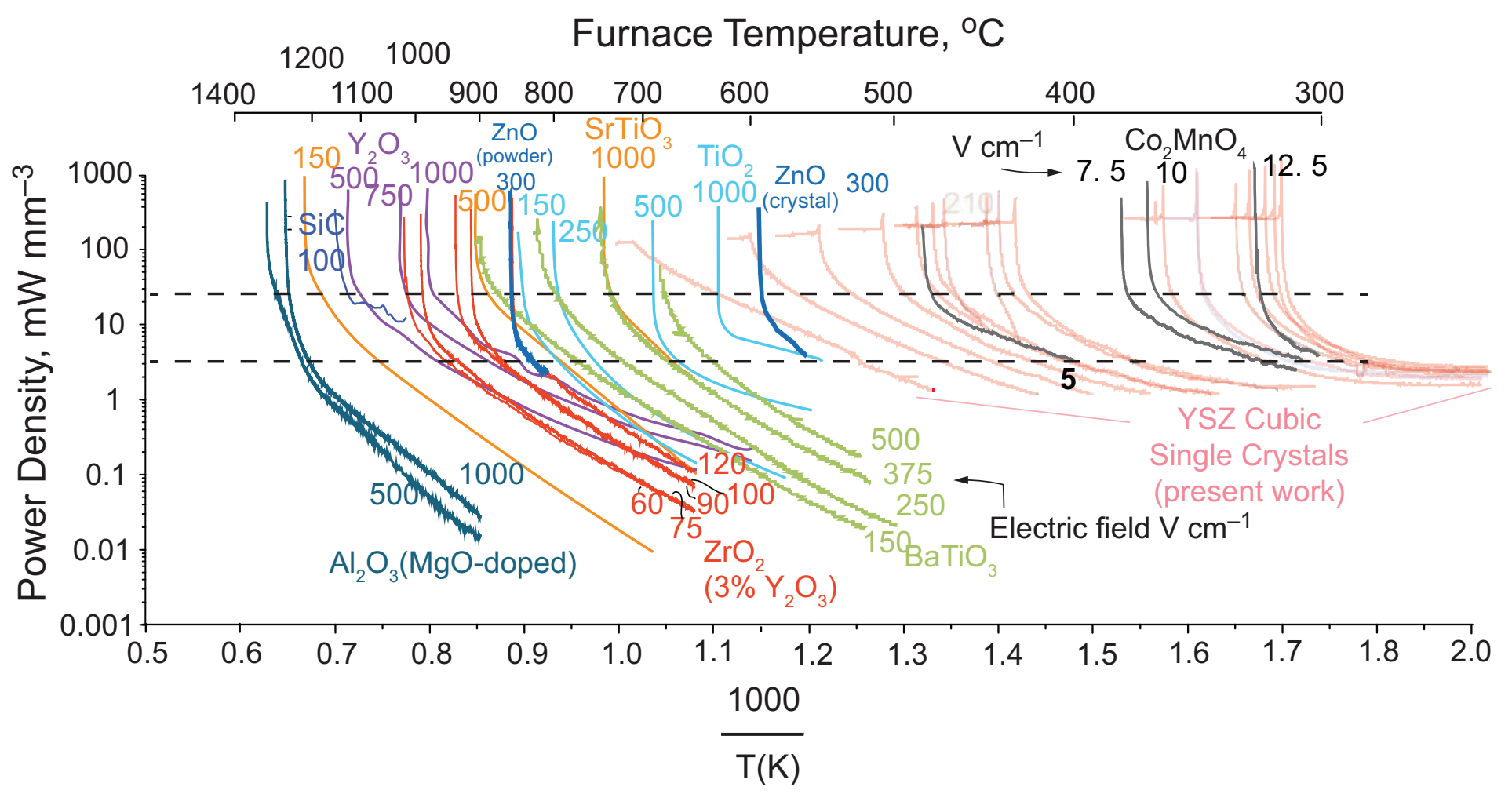

Figure 6. An overlay of the flash transition from earlier work [11] and the current results, which support the result that flash non-linearity occurs within a narrow band of power density. 
Graphical Abstract

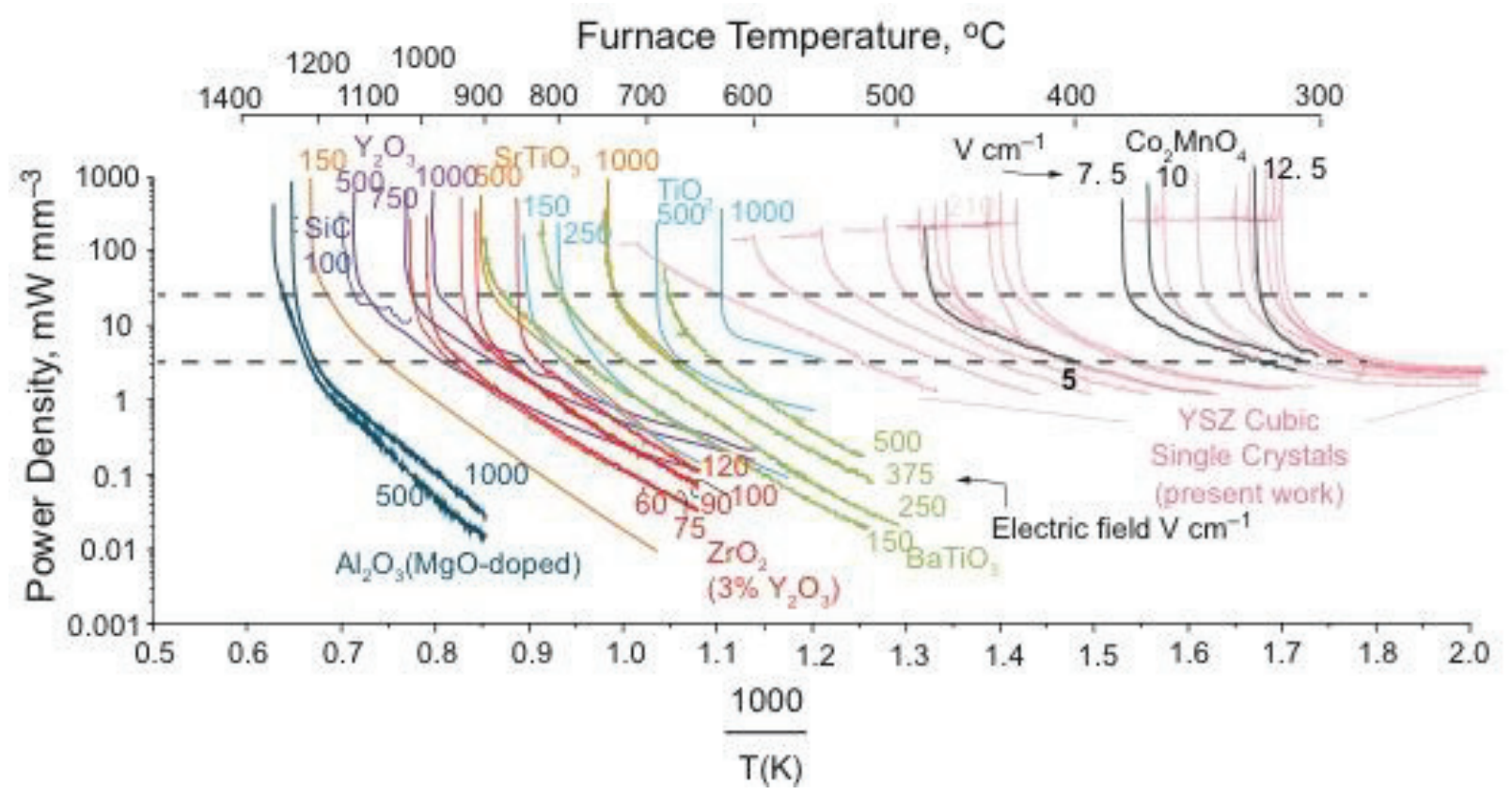

Arrhenius plot of power density showing the transition for various material, and from the present single crystal experiments with cubic zirconia. Note that the transition occurs within a narrow band of the power density suggesting universal behavior.

We have measured the onset of flash in single crystals cubic zirconia, similar to Stage II in flash sintering experiments, at electric fields ranging from $50 \mathrm{~V} \mathrm{~cm}^{-1}$ to $490 \mathrm{~V} \mathrm{~cm}^{-1}$ and temperatures ranging from $\sim 300{ }^{\circ} \mathrm{C}$ to $700{ }^{\circ} \mathrm{C}$. This behavior is compared with results on sintering of porous specimens of 8-10 mol\% cubic zirconia. The single crystals flash at lower temperatures and lower electric fields, which does not support an unalienable role for particle-particle interfaces and grain boundaries in the flash mechanism. The flash onset occurs at similar values of the power density. 